Pacific Journal of Mathematics

ON THE COHOMOLOGY OF KUGA'S FIBER VARIETY 


\title{
ON THE COHOMOLOGY OF KUGA'S FIBER VARIETY
}

\author{
Rita Hall
}

\begin{abstract}
Results of Matsushima and Murakami, and Matsushima and Shimura, are applied to the study of certain subspaces of the cohomology of fiber varieties of Kuga-Satake type. These subspaces are defined in terms of the Kähler and fiber space bigradings of the cohomology of the fiber variety.
\end{abstract}

Let $G$ be a real connected semi-simple Lie group with finite center, and $K$ a maximal compact subgroup of $G$. The quotient space $X=$ $G / K$ is then a symmetric space (of non-compact type). Assume that $X$ is a symmetric domain. Consider a discrete subgroup $\Gamma$ of $G$ such that $\Gamma \backslash G$ is compact and $\Gamma$ has no fixed point in $X$. Take a representation $\rho: G \rightarrow G L(F)$ of $G$ in a real vector space $F$, such that $\rho$ leaves invariant a nonsingular alternating form $\beta$ on $G$. Suppose that $\left.\rho\right|_{\Gamma}$ leaves invariant a lattice $L$ in $F$, and that the form $\beta$ takes integer values on $L \times L$. From the data $\{G, K, X, \Gamma, \rho, F, \beta, L\}$ Kuga has constructed an algebraic variety $V$, fibred by a family of abelian varieties, when $\rho$ satisfies an additional condition.

In this paper the cohomology of the fiber variety $V$ is studied. The cohomology of $V$ is bigraded by the Kähler structure of $V$, and also by the fiber structure of $V$. In $\S 1$ it is shown that the Kähler and fiber bigradings:

$$
\begin{gathered}
H^{r}(V)_{\mathrm{C}}=\sum_{p+q=r} H^{(p, q)}(V) \\
H^{\prime}(V)_{\mathrm{C}}=\sum_{a+b=r} H^{\{a, b\}}(V)_{\mathrm{C}}
\end{gathered}
$$

are compatible, and so lead to a finer decomposition of the cohomology of $V$ :

$$
H^{\prime}(V)_{\mathrm{C}}=\sum H^{a_{1} a_{2} b_{1} b_{2}}
$$

In $\$ 3$ this decomposition is related to the work of Matsushima and Murakami, ([5]) when the representation $\rho$ satisfies a certain 
condition. In $\S 4$ is studied the special case $G=S L(2, \mathbf{R})^{N}$, using results of Matsushima and Shimura [7]. The dimensions of the non-zero $H^{a_{1} a_{2} b_{1} b_{2}}$ are seen to be expressible in terms of the dimensions of spaces of $\Gamma$-automorphic forms. For $N=1$, one obtains

$$
H^{(p, p)}(V)=H^{\{0,2 p\}}(V)_{\mathrm{C}}+H^{\{2,2 p-2\}}(V)_{\mathrm{c}}
$$

This result used in the proof of the Hodge conjecture for the fiber variety $V$ over an algebraic curve associated to an indefinite quaternion algebra over $\mathbf{Q}$.

1. Preliminaries. First recall the construction of the fiber variety $V$. Let $G, K, X$, and $\Gamma$ be as above. The quotient space $U=\Gamma \backslash X$ is then a compact complex manifold, and Kodaira has shown it is a Hodge manifold. Let $B$ be an $N \times N$ integral skew-symmetric matrix with $\operatorname{det} B \neq 0$, and suppose there exists a representation $\rho: G \rightarrow S p(B)=\left\{M \in G L(N R):{ }^{t} M B M=B\right\}$ such that $\left.{ }^{\rho}\right|_{\Gamma}: \Gamma \rightarrow G L(N, Z)$. Denote the representation space $\mathbf{R}^{N}$ by $F$ and the lattice $\mathbf{Z}^{N}$ by $L$. Form the semidirect product $G F$ by defining $(g, u)\left(g^{\prime}, u^{\prime}\right)=\left(g g^{\prime}, \rho(g) u^{\prime}+u\right)$. The group $G F$ acts on $X \times F$ in a natural way, and $\Gamma L$ is a discrete subgroup of $G F$ which acts on $X \times F$ properly discontinuously and without fixed points. The quotient space $V=\Gamma L \backslash X \times F$ is then a compact manifold. The projection $V \rightarrow U$ makes $V$ a fiber bundle over $U$.

Let $(G)$ and $k$ denote the Lie algebras of $G$ and $K$ respectively, and let $(\mathscr{S}=\boldsymbol{k}+\boldsymbol{p}$ be the Cartan decomposition of $(S)$ with respect to $\boldsymbol{k}$. Then one can show there exists an $N \times N$ matrix $S$ such that

1. ${ }^{t} S=S, S>0{ }^{1}$

2. $\left(B^{-1} S\right)^{2}=-1_{N}$

3. $\rho(K) \subset 0(S)=\left\{M \in G L(N, \mathbf{R}):{ }^{t} M S M=S\right\}$

4. ${ }^{t} d \rho(X) S=S d \rho(X), X \in k$

The pair $\{B, S\}$ is called a symplectic pair, and every such pair is conjugate to the pair

$$
\left\{J=\left(\begin{array}{cc}
0 & 1_{N / 2} \\
-1_{N / 2} & 0
\end{array}\right), 1_{N}\right\}
$$

that is, there exists a real $N \times N$ matrix $T$ such that ' $T B T=J$ and ' $T S T=1_{N}$. The mapping $M \rightarrow T^{-1} M T$ gives an isomorphism of $S p(B)$ onto $S p(J)$ and induces an isomorphism

$$
X_{0}=S p(B) / O(S) \cap S p(B) \cong S p(J) / 0\left(1_{N}\right) \cap S p(J) .
$$


The latter space is identified with the Siegel space $\mathfrak{h}^{N / 2}=$ $\{Z \in M(N / 2), C):{ }^{\prime} Z=Z$ and $\left.\operatorname{Im} Z>0\right\}$ by

$$
S p(J) \ni\left(\begin{array}{cc}
A & B \\
C & D
\end{array}\right) \rightarrow(A i+B)(C i+D)^{-1} \in \mathfrak{h}^{N / 2} .
$$

Of the two possible complex structures on $X_{0}$, we give the one making the mapping $X_{0} \rightarrow \mathfrak{h}^{N / 2}$ holomorphic.

Assume that the mapping $\tau: X \rightarrow X_{0}$ induced by $\rho: G \rightarrow S p(B)$ is holomorphic. This assumption makes it possible to define a $G F$ invariant complex structure on $X \times F$. Let $\rho^{\prime}: G \stackrel{\rho}{\rightarrow} S p(B) \stackrel{a}{\rightarrow} S p(J)$ and let $\tau^{\prime}: X \rightarrow \mathfrak{h}^{N / 2}$ be the induced mapping. Let $\xi(u)$ be the coordinate vector of $u \in F=\mathbf{R}^{N}$ with respect to the standard basis of $\mathbf{R}^{N}$, and let $\xi^{\prime}(u)=T^{-1} \xi(u)$. Define

$$
Z(x, u)=\left(\begin{array}{c}
Z^{\perp}(x, u) \\
\vdots \\
Z^{N / 2}(x, u)
\end{array}\right)=\left(\tau^{\prime}(x), 1_{N / 2}\right) J \xi^{\prime}(u) .
$$

If $w^{1}(x), \cdots, w^{n}(x)$ is a system of complex coordinates for $X$, then the system $w^{1}(x), \cdots, w^{n}(x), Z^{1}(x, u), \cdots, Z^{N / 2}(x, u)$ gives complex coordinates for $(x, u) \in X \times F$. With respect to this complex structure on $X \times F$, the action of $G F$ is holomorphic. Give $V$ the induced complex structure.

Let $x=g K$. Then $A(x)={ }^{\prime} \rho(g)^{-1} S \rho(g)^{-1}$ and $J(x)=B^{-1} A(x)$ are well-defined, and $J(x)$ induces the complex structure on the fiber over $\Gamma x$ in $U$. If $d s_{0}^{2}$ is a Hodge metric on $U$, then the metric $d s^{2}=$ $d s_{0}^{2}+{ }^{t} d \xi(u) A(x) d \xi(u)$ makes $V$ a Hodge manifold.

Let $\Omega^{r}(X)$ be the space of real valued $r$-forms on $X$. Let $\Omega^{\prime}(X, \Gamma, \rho)=\left\{\omega \in F \otimes \Omega^{r}(X): \omega \circ \gamma=\rho(\gamma) \omega\right\}$. The exterior differentiation $d$ on $\Omega^{*}(X)$ gives a coboundary operator $1 \otimes d$ on $\Omega^{*}(X, \Gamma, \rho)$. Let $H^{\prime}(X, \Gamma, \rho)$ be the $r$ th cohomology group of the complex $\left(\Omega^{*}(X, \Gamma, \rho), 1 \otimes d\right)$. Given a suitable inner product $(,)_{F}$ on $F$, the notion of harmonic element of $\Omega^{r}(X, \Gamma, \rho)$ can be defined. Let $\mathscr{H}^{a}(X, \Gamma, \rho)=\left\{\omega \in \Omega^{r}(X, \Gamma, \rho): \omega\right.$ is harmonic $\} . \mathscr{H}^{a}(X, \Gamma, \rho)$ and $H^{a}(X, \Gamma, \rho)$ are naturally isomorphic.

Identify $\Lambda^{b}(F)^{*}$, the dual to the Grassmanian $\Lambda^{b}(F)$, with $\Lambda^{b}\left(F^{*}\right)$. Choose an ordered basis $\left\{\xi^{D_{i}}: i=1, \cdots,\left(\begin{array}{c}N \\ b\end{array}\right)\right\}$ of $\Lambda^{b}(F)^{*}$, such that each $\xi^{D_{i}}$ is of the form $\xi^{D}=\xi^{D_{1}} \wedge \cdots \wedge \xi^{d_{b}}$ where $D=\left(d_{1}, \cdots, d_{b}\right)$ and $\left\{\xi^{1}, \cdots, \xi^{N}\right\}$ is the usual coordinate system on $F=\mathbf{R}_{N}$. This notation will also be used for differential forms; e.g., $d \xi^{D}$ for $d \xi^{d_{1}} \wedge \cdots \wedge d \xi^{d_{b}}$. Let $\Lambda^{b}$ be the natural representation of $G L(F)$ on $\Lambda^{b}(F)$, and let $\rho^{(b)}=\left(\Lambda^{b} \circ \rho\right)^{*}$ be the representation of $G$ on $\Lambda^{b}(F)^{*}$, contragredient to $\Lambda^{b} \circ \rho$. The symmetric positive definite matrix $S$ given 
above can be used to define a suitable inner product on $\Lambda^{b}(F)^{*}$, so that $\mathscr{H}^{a}\left(X, \Gamma, \rho^{(b)}\right)$ is defined. (The condition of suitability is that, with respect to $(,)_{F}, \rho(X)$ is skew-symmetric if $X \in k$ and symmetric if $X \in p$.)

Let $\mathscr{H}^{r}(V)$ be the space of real harmonic forms on the Kähler manifold $V$, and let $H^{r}(V)$ be the $r$ th real de Rham cohomology group of $V . \quad H^{r}(V)$ and $\mathscr{H}^{r}(V)$ are naturally isomorphic; this isomorphism will be referred to as the Hodge isomorphism. One can define a mapping of $\mathscr{H}^{a}\left(X, \Gamma, \rho^{(b)}\right)$ into $\mathscr{H}^{a+b}(V)$ by

$$
\mathscr{H}^{a}\left(X, \Gamma, \rho^{(b)}\right) \ni \hat{\omega}(x) \rightarrow^{t} \hat{\omega}(x) \wedge\left[\begin{array}{c}
d \xi^{D_{1}}(u) \\
\vdots \\
d \xi^{D}\left(\begin{array}{c}
N \\
b
\end{array}\right)(u)
\end{array}\right]=\omega(x, u) \in \mathscr{H}^{a+b}(V)
$$

(Here, differential forms on $V$ are considered to be $\Gamma L$-invariant forms on $X \times F$.) Let $\mathscr{H}^{\{a, b\}}(V)$ be the image of this mapping. Then the space $H^{\{a, b\}}(V)$ occurring in the decomposition $(f)$ is the image of $\mathscr{H}^{\{a, b\}}(V)$ under the Hodge isomorphism. See [3] for details and proofs.

2. In this section the decomposition

$$
\mathscr{H}^{\{a, b\}}(V)_{\mathrm{c}}=\sum_{\substack{a_{1}+a_{2}=a \\ b_{1}+b_{2}=b}} \mathscr{H}^{a_{1} a_{2} b_{1} b_{2}}
$$

is obtained. $\quad\left(\mathscr{H}^{\{a, b\}}(V)_{\mathbf{C}}\right.$ is the complexification $\mathscr{H}^{\{a, b\}}(V) \otimes \mathbf{C}$ of the real vector space $\mathscr{H}^{\{a, b\}}(V)$.) Rewrite equation (1) of $\S 1$ as

$$
\left(\begin{array}{l}
z \\
\bar{z}
\end{array}\right)=\left(\begin{array}{cc}
-1 & \tau^{\prime} \\
-1 & \bar{\tau}^{\prime}
\end{array}\right) \xi^{\prime}
$$

By taking differentials obtain $(1,0)$-forms $u^{1}(x, u), \cdots, u^{N / 2}(x, u)$ defined by the equation

$$
\left(\begin{array}{l}
u \\
\bar{u}
\end{array}\right)=\left(\begin{array}{ll}
-1 & \tau^{\prime} \\
-1 & \bar{\tau}^{\prime}
\end{array}\right) d \xi^{\prime}=\left(\begin{array}{ll}
d z & -d \tau^{\prime} \cdot \xi^{\prime \prime} \\
d \tilde{z} & -d \bar{\tau}^{\prime} \cdot \xi^{\prime \prime}
\end{array}\right)
$$

where

$$
\xi^{\prime \prime}=\left(\begin{array}{c}
\xi^{\prime N / 2+1} \\
\vdots \\
\xi^{\prime N}
\end{array}\right)
$$

For $D=\left(d_{1}, \cdots, d_{b}\right)$, put $|2 D|=b$. 
Definition. Let $\omega \in \mathscr{H}^{\{a, b\}}(V)_{\mathrm{c}} . \quad \omega$ is of type $a_{1} a_{2} b_{1} b_{2}$ if

$$
\omega(x, u)=\Sigma \psi_{A_{1} A_{2} B_{1} B_{2}} d w^{A_{1}} \wedge d \bar{w}^{A_{2}} \wedge u^{B_{1}} \wedge \bar{u}^{B_{2}}
$$

where $\psi_{A_{1} A_{2} B_{1} B_{2}}=0$ unless $\left|A_{i}\right|=a_{i}$ and $\left|B_{i}\right|=b_{i}, i=1,2$. The terminology " $a_{1} a_{2} b_{1} b_{2}$-component of $\omega \in \mathscr{H}^{\{a, b\}}(V)_{\mathrm{c}}$ " will be used, whether or not that component is harmonic. Let

$$
\mathscr{H}^{a_{1} a_{2} b_{1} b_{2}}=\left\{\omega \in \mathscr{H}^{\left\{a_{1} b\right\}}(V)_{\mathrm{C}}: \omega \text { is of type } a_{1} a_{2} b_{1} b_{2}\right\}
$$

Several definitions are needed. Let $\mathbb{F}_{c}$ be the complexification of the Lie algebra of $G$. The representation $\rho: G \rightarrow G L(F)$ induces a representation $\rho_{\mathrm{C}}: G \rightarrow G L(F) \subset G L\left(F_{\mathrm{C}}\right)$ and a Lie algebra representation $\rho=d \rho_{C}:\left(\mathbb{S}_{\mathrm{C}} \rightarrow G L\left(F_{\mathrm{C}}\right)\right.$. The isomorphism (2) of $\S 1$ given by $\hat{\omega} \rightarrow \omega$ extends to a $\mathbf{C}$-linear isomorphism of the complexifications

$$
\mathscr{H}^{a}\left(X, \Gamma, \rho^{(b)}\right)_{\mathrm{c}} \cong \mathscr{H}^{\{a, b\}}(V)_{\mathrm{c}}
$$

An invariant complex structure on $X_{0}=S p(B) / 0(S) \cap S p(B)$ is determined by the element $Z_{0}=-1 / 2 B^{-1} S$ belonging to the center of $k_{0}$, the Lie algebra of $0(S) \cap S p(B)$; and the invariant complex structure on $X$ is determined by an element $Z$ belonging to the center of $\boldsymbol{k}$.

Let $\mathscr{S}_{\mathrm{c}}=\boldsymbol{k}_{\mathrm{c}}+\boldsymbol{p}_{+}+\boldsymbol{p}_{-}$be the decomposition of $\mathscr{S}_{\mathrm{c}}$ into (respectively) the $0,+i$, and $-i$-eigenspaces of $\operatorname{ad} Z$. Then $\boldsymbol{k}_{\mathrm{c}}$ is orthogonal to $\boldsymbol{p}_{\mathrm{C}}=\boldsymbol{p}_{+}+\boldsymbol{p}_{-}$with orespect to the Killing form $K$, and there exists a basis $Y_{1}, \cdots, Y_{s}$ of $k_{c}$ and a basis $X_{1}, \cdots, X_{n}$ of $p_{+}$such that

$$
\begin{array}{cl}
K\left(Y_{a}, Y_{b}\right)=-\delta_{a b} & a, b=1, \cdots, s \\
K\left(X_{\imath}, \bar{X}_{\jmath}\right)=\delta_{y} & i, j=1, \cdots, n .
\end{array}
$$

Put $\boldsymbol{X}_{\bar{I}}=\overline{\boldsymbol{X}}_{1} \in \boldsymbol{p}_{-}=\overline{\boldsymbol{p}}_{+}$.

To $\omega \in \mathscr{H}^{\{a, b\}}(V)_{c}$ one can associate a coordinate function $\omega^{0}(g)$ taking values in $\Lambda^{b}\left(F_{\mathrm{c}}\right)^{*} \otimes \Lambda^{a}\left(p_{\mathrm{c}}\right)^{*}$. First identify $\Lambda^{a}\left(\boldsymbol{p}_{\mathrm{c}}\right)^{*}$ with $\Lambda^{a}\left(\boldsymbol{p}_{\mathrm{c}}\right)$ by means of the Killing form. Let $v: G \rightarrow X$ be the projection. For $I=\left(i_{1}, \cdots, i_{a_{1}}\right), J=\left(j_{1}, \cdots, j_{a_{2}}\right)$, and $a_{1}+a_{2}=a$, define

$$
\omega_{\bar{I}}(g)=\rho^{(b)}\left(g^{-1}\right) v^{*} \hat{\omega}_{g}\left(X_{t_{1}}, \cdots, X_{t a 1}, X_{\overline{j 1}}, \cdots, X_{\bar{j}_{2}}\right)
$$

and let

$$
\omega^{o}(g)=\Sigma \omega_{I \bar{I}}(g) X_{\bar{I}} \Lambda X_{J}
$$

Let $C$ be the Casimir operator of $G$, 


$$
C=\sum_{k=1}^{n} X_{k} X_{\bar{k}}+X_{\bar{k}} X_{k}-\sum_{a=1}^{s} Y_{a}^{2}
$$

and let $\rho^{(b)}(C)$ be the Casimir matrix of the representation $\rho^{(b)}$. Then one can show

Proposition 1. $\omega \in \mathscr{H}^{\{a, b\}}(V)_{\mathbf{c}} \Leftrightarrow$

(i) $\omega^{\circ}(\gamma g)=\omega^{\circ}(g) \forall \gamma \in \Gamma, g \in G$

(ii) $\omega^{o}\left(g k^{-1}\right)=\rho^{(b)}(k) \otimes \Lambda^{a} o A d(k) \forall g \in G, k \in K$

(iii) $C \omega^{\circ}=\rho^{(b)}(C) \otimes 1 \omega^{\circ}$

(for the proof, cf. [3]). (Ad is the adjoint representation of $K$ on $\mathrm{p}_{\mathrm{c}}$ ) Let $V^{b_{1} b_{2}}$ be the $\left(b_{2}-b_{1}\right) i / 2$-eigenvalue of $d \Lambda^{b}\left(\begin{array}{cc}i / 2 & 0 \\ 0 & -i / 2\end{array}\right)$ in $C^{\left(b_{b}\right.}$. Recall there exists $T \in G L(N, \mathbf{R})$ such that ${ }^{t} T B T=J$ and $' T S T=1$, and that $\rho^{\prime}=T^{-1} \rho T$. Let $L=\frac{1}{\sqrt{2}}\left(\begin{array}{cc}i & 1 \\ -i & 1\end{array}\right)$, and let $L_{0}=T L^{-1}$. Then

$$
Z_{0}=-\frac{1}{2} B^{-1} S=L_{0}\left(\begin{array}{cc}
i / 2 & 0 \\
0 & -i / 2
\end{array}\right) L_{0}^{-1}
$$

So that $F^{b_{1} b_{2}}=\Lambda^{b}\left(L_{0}\right)^{*}\left(V^{b_{1} b_{2}}\right)$ is the $\left(b_{2}-b_{1}\right) i / 2$-eigenspace of $d \Lambda^{b}\left(Z_{0}\right)^{*}$. Now take the $a_{1} a_{2} b_{1} b_{2}$-component $\eta$ of $\omega \in \mathscr{H}^{\{a, b\}}(V)_{c}$ and make the $F$-valued form $\hat{\eta}$ and $F$-valued functions $\eta_{\bar{L}}$ as in (4). Then

Proposition 2. $\eta_{\bar{L}}$ takes values in $F^{b_{1} b_{2}}$ and

$$
\eta_{I \bar{J}}=0 \text { unless }|I|=a_{1} \text { and }|J|=a_{2} .
$$

Proof. Since $\hat{\eta}$ is of type $\left(a_{1}, a_{2}\right)$ and the action of $\operatorname{ad}(Z)$ on $\rho$ determines, via the projection $G \rightarrow X$, the complex structure on $X$, one sees that $\eta_{\bar{L}}=0$ unless $|I|=a_{1}$ and $|J|=a_{2}$. Since

$$
\left(\begin{array}{c}
u \\
\bar{u}
\end{array}\right)=\left(\begin{array}{cc}
-1 & \tau^{\prime} \\
-1 & \bar{\tau}^{\prime}
\end{array}\right) T^{-1} d \xi \quad \text { and } \quad \eta={ }^{\prime} \hat{\eta} \wedge\left(\begin{array}{c}
\vdots \\
d \xi^{D_{\imath}} \\
\vdots
\end{array}\right)
$$

one sees that the form

$$
\zeta=\wedge^{b}\left(\left(\begin{array}{cc}
-1 & \tau^{\prime} \\
-1 & \bar{\tau}^{\prime}
\end{array}\right) T^{-1}\right)^{*} \hat{\eta}
$$

takes values in $V^{b_{1} b_{2}}$. Now

$$
\eta_{\bar{L}}(g)=\Lambda^{b}\left(T \rho^{\prime}\left(g^{-1}\right)\left(\begin{array}{cc}
-1 & \tau^{\prime} \\
-1 & \bar{\tau}^{\prime}
\end{array}\right)^{-1}\right)^{*} v^{*} \zeta_{g}\left(X_{i 1}, \cdots, X_{\overline{I a}}\right)
$$


Let $\rho^{\prime}(g)=\left(\begin{array}{cc}A & B \\ C & D\end{array}\right), \tau^{\prime}=\tau^{\prime}(g k)$ and $j={ }^{\prime}(C i+D)^{-1}$. Then using the relations

$$
\begin{gathered}
\operatorname{Im} \tau^{\prime}=j^{-1} t_{j}, \\
\rho^{\prime}(g)\left(\begin{array}{cc}
i & -i \\
1 & 1
\end{array}\right)=\left(\begin{array}{cc}
\tau^{\prime} & \bar{\tau}^{\prime} \\
1 & 1
\end{array}\right)\left(\begin{array}{cc}
t_{1}^{-1} & 0 \\
0 & t_{\sigma^{-1}}
\end{array}\right),
\end{gathered}
$$

one finds

$$
T \rho^{\prime}(g)^{-1}\left(\begin{array}{cc}
-1 & \tau^{\prime} \\
-1 & \bar{\tau}^{\prime}
\end{array}\right)^{-1}=L_{0}\left(\begin{array}{cc}
\sqrt{-2} j^{-1} & 0 \\
0 & \sqrt{-2} \bar{j}^{-1}
\end{array}\right)
$$

Since $V^{b_{1} b_{2}}$ is $\Lambda^{b}\left(\begin{array}{cc}\sqrt{-2} j^{-1} & 0 \\ 0 & \sqrt{-2 \bar{j}^{-1}}\end{array}\right)$-invariant, $\eta_{L \bar{J}} \in \Lambda^{b}\left(L_{0}\right)^{*}\left(V^{b_{1} b_{2}}\right)=$ $F^{b_{1} b_{2}}$, proving the proposition.

Since the representation $\rho$ induces a holomorphic mapping $\tau: X \rightarrow X_{0}$, we have $\left.\rho\right|_{p_{+}}: p_{+} \rightarrow p_{0_{+}}$and $\left.\rho\right|_{p_{-}}: p_{-} \rightarrow p_{0_{-}}$, where $p_{0_{+}}\left(\operatorname{resp} p_{0_{-}}\right)$ is the $+i$ (resp. $-i$ )-eigenspace of $\operatorname{ad} Z_{0}$. Hence

$$
\rho^{(b)}\left(X_{k}\right): F^{b_{1} b_{2}} \rightarrow F^{b_{1}-1, b_{2}+1}
$$

and

$$
\rho^{(b)}\left(X_{\bar{k}}\right): F^{b_{1} b_{2}} \rightarrow F^{b_{1}+1, b_{1}-1}
$$

From (5), one sees that $F^{b_{1} b_{2}}$ is invariant under $\rho^{(b)}(C)$. Furthermore, for any $k \in K, \quad \rho^{(b)}(k) \otimes \Lambda^{a} \circ A d(k)$ sends $F^{b_{1} b_{2}} \otimes \Lambda^{a_{1}}\left(\boldsymbol{p}_{-}\right) \otimes \Lambda^{a_{2}}\left(\boldsymbol{p}_{+}\right)$to itself. This implies that conditions (i) thru (iii) of Proposition 1 are satisfied by the coordinate function

$$
\eta^{o}=\Sigma \eta_{\bar{L}}(g) X_{\bar{I}} \wedge X_{J}
$$

hence the $a_{1} a_{2} b_{1} b_{2}$-component of a harmonic form is harmonic. In summary:

Proposition 3. Let $\omega \in \mathscr{H}^{\{a, b\}}(V)_{\text {c. Then }}$. The $\omega \in \mathscr{H}^{a_{1} a_{2} b_{1} b_{2}} \Leftrightarrow \omega^{o}$ takes values in $F^{b_{1} b_{2}} \otimes \Lambda^{a_{1}}\left(\boldsymbol{p}_{-}\right) \otimes \Lambda^{a_{2}}\left(\boldsymbol{p}_{+}\right)$.

The following theorem is now immediate.

THEOREM 1.

$$
\mathscr{H}^{\{a, b\}}(V)_{\mathbf{C}}=\sum_{\substack{a_{1}+a_{2}=a \\ b_{1}+b_{2}=b}} \mathscr{H}^{a_{1} a_{2} b_{1} b_{2}}
$$




$$
\mathscr{H}^{(p, q)}(V)=\sum_{\substack{a_{1}+b_{1}=p \\ a_{2}+b_{2}=p}} \mathscr{H}^{a_{1} a_{2} b_{1} b_{2}}
$$

3. In this section assume that the representation $\rho$ satisfies the condition

$$
\rho(Z)=Z_{0}
$$

This assumption allows the application of some results of Matsushima and Murakami ([5]) to the study of the spaces $\mathscr{H}^{a_{1} a_{2} b_{1} b_{2}}$.

Let $\Sigma$ be the root system of $\mathscr{S}_{c}$ with respect to a Cartan subalgebra contained in $\boldsymbol{k}_{\mathrm{c}}$. Choose an ordering of the roots such that the roots belonging to $p_{+}$are positive. Let $\Sigma^{+}$(resp., $\Sigma^{-}$) denote the set of positive (resp. negative) roots. Let $\psi$ denote the set of positive noncompact roots and let $\theta$ denote the set of positive compact roots. Let $W$ be the Weyl group of $\mathbb{G}_{\mathrm{C}}$, and let $W_{1}$ be the Weyl group of $\boldsymbol{k}_{\mathrm{C}}$, considered as a subgroup of $W$. Let $R_{1}$ be the involution in $W_{1}$ satisfying $R_{1} \theta=$ $-\theta$. For $T \in W$ put $\phi_{T}=T\left(\Sigma^{-}\right) \cap \Sigma^{+}$, and put $\left\langle\phi_{T}\right\rangle=\Sigma_{\alpha \in \phi_{T}} \alpha$. Let $\delta=\frac{1}{2} \Sigma_{\alpha \in \Sigma_{+}} \alpha$. Then $\left\langle\phi_{T}\right\rangle=\delta-T \delta$. Let $W^{\prime}=\left\{T \in W: \phi_{T} \subset \psi\right\}$. Let $n(T)=\operatorname{Card}(\phi(T))$. There is a direct sum decomposition

$$
\Lambda^{a_{1}}\left(\boldsymbol{p}_{-}\right) \otimes \Lambda^{a_{2}}\left(\boldsymbol{p}_{+}\right)=\sum_{\substack{n(S)=a_{1} \\ n(T)=a_{2}}} P^{-\left\langle\phi_{s}\right\rangle} \otimes Q_{\left(\phi_{T}\right\rangle}
$$

of $\Lambda^{a_{1}}\left(\boldsymbol{p}_{-}\right) \otimes \Lambda^{a_{2}}\left(\boldsymbol{p}_{+}\right)$into a sum of $\boldsymbol{k}_{\mathbf{c}^{-}}$-invariant submodules (with respect to the representation $\left.\operatorname{ad}_{-}^{a_{1}} \otimes \mathrm{ad}_{+}^{a_{2}}\right)$; the submodule $P^{-\left\langle\phi_{s}\right\rangle}\left(\right.$ resp. $\left.Q_{\left\langle\phi_{T}\right)}\right)$ is the (unique) irreducible $\boldsymbol{k}_{\mathrm{C}}$-submodule of $\Lambda^{a_{1}}\left(\boldsymbol{p}_{-}\right)$(resp. $\Lambda^{a_{2}}\left(\boldsymbol{p}_{+}\right)$) having highest weight $-\left\langle\phi_{S}\right\rangle$ (resp. lowest weight $\left\langle\phi_{T}\right\rangle$ ).

Let $F$ now be a complex vector space and $\rho^{\Lambda}: G \rightarrow G L(F)$ an irreducible representation of $G$ with highest weight $\Lambda$. Then the spaces $H^{a}\left(X, \Gamma, \rho^{\Lambda}\right)$, and, when a suitable hermitian inner product is given on $F$, the spaces $\mathscr{H}^{a}\left(X, \Gamma, \rho^{\Lambda}\right)$ of harmonic $F$-valued forms on $V$ can be defined. To $\omega \in \mathscr{H}^{a}\left(X, \Gamma, \rho^{\Lambda}\right)$ one can associate a coordinate vector $\omega^{\circ}$ as in $\$ 1$. For each $g \in G, \omega^{\circ}(g)$ determines a "harmonic representative" of a Lie algebra cohomology class. (See [5] for explanation and details.)

Now consider $F$ to be a $\boldsymbol{k}_{\mathbf{c}}$-module by restricting the differential of the representation $\rho^{\Lambda}$ to the subalgebra $\boldsymbol{k}_{\mathrm{c}}$ of $\mathbb{G}_{\mathrm{c}}$. Let $F^{\mu}$ be irreducible $\boldsymbol{k}_{\mathrm{c}}$-submodule of $F$ with highest weight $\mu$. Let $\mathfrak{h}(\Lambda, \mu, S, T)=$ $\left\{C \in F^{\mu} \otimes P^{-\left\langle\phi_{s}\right\rangle} \otimes Q_{\langle\phi\rangle}\right\rangle: C$ is "harmonic" $\}$. Let $\Lambda^{\prime}$ denote the lowest weight of the representation $\rho^{\Lambda}$. In [5], the following lemma is proved:

LEMMA 1. $\mathfrak{h}(\Lambda, \mu, S, T)=0$ unless $\mu=S \Lambda=R_{1}^{-1} T \Lambda^{\prime}$. 
Identify $\mathscr{H}^{a}\left(X, \Gamma, \rho^{(b)}\right)_{c}$ and $\mathscr{H}^{a}\left(X, \Gamma, \rho_{\mathrm{c}}{ }^{(b)}\right)$, and fix a direct sum decomposition

$$
\Lambda^{b}\left(F_{\mathrm{c}}\right)^{*}=\Sigma \mathrm{F}_{1}(\Lambda)
$$

of $\Lambda^{b}\left(F_{\mathbf{C}}\right)^{*}$ into a direct sum of irreducible $\mathscr{S}_{\mathrm{C}}$-submodules such that $\left.\rho^{(b)}\right|_{F(\Lambda)} \sim \rho^{\Lambda}, \rho^{\Lambda}$ being irreducible, with highest weight $\Lambda$. By the assumption (6), $\rho^{(b)}(Z)=d \Lambda^{b}\left(Z_{0}\right)^{*}$, and so

$$
F^{b_{1} b_{2}}=\Sigma F_{1}^{b_{1} b_{2}}(\Lambda)
$$

where $F_{1}^{b_{1} b_{2}}(\Lambda)=F_{1}(\Lambda) \cap F^{b_{1} b_{2}}$. One can consider $F_{1}(\Lambda)$ as a representation space of $K_{\mathrm{c}}$. Then one obtains a direct sum decomposition

$$
F_{i}(\Lambda)=\Sigma F_{\imath \jmath}(\Lambda, \mu)
$$

of $F_{1}(\Lambda)$ into irreducible $\boldsymbol{k}_{\mathrm{C}}$-submodules $F_{y}(\Lambda, \mu), \mu$ being the highest weight of the representation on $F_{i l}(\Lambda, \mu)$. Now define, for $S, T \in W^{\prime}$ and fixed $\Lambda$ and $\mu$ as above

$$
\begin{aligned}
\mathscr{H}^{\{a, b\}}(\Lambda, \mu, S, T,)= & \left\{\omega \in \mathscr{H}^{\{a, b\}}(V)_{\mathrm{c}}: \omega^{o}\right. \text { takes values } \\
& \text { in } \Sigma F_{y \jmath}(\Lambda, \mu) \otimes P^{-\left\langle\phi_{s}\right\rangle} \otimes Q_{\left\langle\phi_{T}\right\}} .
\end{aligned}
$$

Since $F_{y \jmath}(\Lambda, \mu) \otimes P^{-\left\langle\phi_{s}\right\rangle} \otimes Q_{\left\langle\phi_{T}\right\rangle}$ is $\rho^{(b)}(C)^{-}$and $\rho^{(b)}(k) \otimes \Lambda^{a} \circ A d(k)$ invariant,

$$
\mathscr{H}^{\{a, b\}}(V)_{\mathbf{c}}=\sum_{n(S)+n(T)=a} \mathscr{H}^{\{a, b\}}(\Lambda, \mu, S, T) .
$$

The subspace

$$
V_{\mathrm{l}}(\Lambda)=\left\{\omega \in \mathscr{H}^{\{a, b\}}(V)_{\mathrm{c}}: \omega^{o} \text { takes values in } F_{\mathrm{l}}(\Lambda) \otimes \Lambda^{a}\left(\boldsymbol{p}_{\mathrm{c}}\right)^{*}\right\}
$$

is isomorphic to $\mathscr{H}^{a}\left(X, \Gamma, \rho^{\Lambda}\right)_{\mathrm{c}}$. If $\omega \in V_{1}(\Lambda) \cap \mid \mathscr{H}^{\{a, b\}}(\Lambda, \mu, S, T)$, then, letting $F=F_{i}(\Lambda), \omega^{o}(g) \in \mathfrak{h}(\Lambda, \mu, S, T)$, and so Lemma 1 implies that this intersection is $\{0\}$ unless $\mu=S \Lambda=R_{1}^{-1} T \Lambda^{\prime}$, where $\Lambda^{\prime}$ is the lowest weight of $\rho^{\Lambda}$. Let $d(\Lambda, \mu, S, T)=\operatorname{dim} V_{\imath}(\Lambda) \cap \mathscr{H}^{\{a, b\}}(\Lambda, \mu, S, T)$. (This dimension is independent of $i$.)

Proposition 4. Let $a(\Lambda, b)$ denote the multiplicity of the irreducible representation $\rho^{\Lambda}$ in the representation $\rho_{\mathrm{c}}^{(b)}$, and let $S, T \in W^{\prime}$. Then $R_{1}^{-1} T \Lambda^{\prime}$

(1) $\mathscr{H}^{\{a, b\}}(\Lambda, \mu, S, T)=0$ unless $n(S)+n(T)=a$ and $\mu=S \Lambda=$ 
(2) $\operatorname{dim} \mathscr{H}^{\{a, b\}}(\Lambda, \mu, S, T)=a(\Lambda, b) d(\Lambda, \mu, S, T)$

(3) If $\mathscr{H}^{\{a, b\}}(\Lambda, \mu, S, T) \neq 0$, then $\mathscr{H}^{\{a, b\}}(\Lambda, \mu, S, T) \subset H^{a_{1} a_{2} b_{1} b_{2}}$ where $a_{1}=n(S), a_{2}=n(T)$ and $b_{1}$ and $b_{2}$ aré determined by the conditions: $b_{1}+b_{2}=b$ and $\left(b_{2}-b_{1}\right) i / 2=\mu(Z)$.

(4) $\mathscr{H}^{a_{1} a_{2} b_{1} b_{2}}=\Sigma \mathscr{H}^{\{a, b\}}(\Lambda, \mu, S, T)$, the summation being taken over $S, T \in W^{\prime}$ such that $n(S)=a_{1}, n(T)=a_{2}$ and $\mu$ satisfying the conditions of (1) and (3).

Proof. (1) and (2) follow form (10), (12) and the remarks preceding the proposition, and (4) follows from (3), (11), and Theorem 1 . Now, (since $Z$ belongs to the center of $\left.k_{c}\right) F_{1 y}(\Lambda, \mu)$ belongs to the $\mu(Z)$ eigenspace of $\rho^{(b)}(Z)$. By assumption, $\rho^{(b)}(Z)=d \Lambda^{b}\left(Z_{0}\right)^{*}$ so $F_{1 y}(\Lambda, \mu) \subset F^{b_{1} b_{2}}$, where $b_{1}+b_{2}=b$ and $\left(b_{2}-b_{1}\right) i / 2=\mu(Z)$. Use (7) and Proposition 3 to complete the proof of 3 .

4. Now consider the special case where $V$ is a fiber variety associated to $G=S L(2, \mathbf{R})^{N}$ (= the direct product of $N$ copies of $S L(2, \mathbf{R})), K=S O(2, \mathbf{R})^{N}, \rho: G \rightarrow S p(B)$ a representation equivalent to $h$ copies of the identity representation, and $\Gamma$ an irreducible subgroup of $G$ satisfying the conditions given before. The quotient $X=G / K$ may be identified with $N$ copies of the upper half plane.

Denote by $Z^{k}$ the complex coordinate on the $k$-th factor, and let $z=\left(z^{1}, \cdots, z^{N}\right)$. The irreducible representations of $G$ are of the form $\rho_{(m)}=\rho_{m_{1}} \otimes \cdots \otimes \rho_{m_{N}}$ where $\rho_{m_{k}}$ is the symmetric tensor representation of $S L(2, \mathbf{R})$ of degree $m_{k}+1$. Denote the representation space of $\rho_{(m)}$ by $F_{(m)}$, and the highest weight of $\rho_{(m)}$ by $\Lambda_{(m)}$. In [6], the subspaces $\mathfrak{h}\left(k_{1}, \cdots, k_{p} ; l_{1}, \cdots, l_{q} ; \rho_{(m)}\right)$ of $\mathscr{H}^{p+q}\left(X, \Gamma, \rho_{(m)}\right)$ are studied. $\mathfrak{h}\left(k_{1}, \cdots, k_{p} ; l_{1}, \cdots, l_{q} ; \rho_{(m)}\right)$ contains all the harmonic $\left.F_{(m)}\right)^{-}$ valued forms

$$
\omega_{z}=f(z) d z^{k_{1}} \wedge \cdots \wedge d z^{k_{p}} \wedge d \bar{z}^{l_{1}} \wedge \cdots d \bar{z}^{I_{q}}
$$

which satisfy the condition: $(\omega \circ \gamma)_{z}=\rho_{(m)}(\gamma) \omega_{z}$ for all $\gamma \in \Gamma$. It is proved in [6] that $\operatorname{dim} \mathfrak{h}\left(k_{1}, \cdots, k_{p} ; l_{1}, \cdots, l_{q} ; \rho_{(m)}\right)=0$ unless $(m)=\left(m_{1}, \cdots, m_{N}\right)=(0, \cdots, 0), p=q \quad$ and $\quad\left\{k_{1}, \cdots, k_{p}\right\}=\left\{l_{1}, \cdots, l_{q}\right\}$; or unless $\left\{k_{1}, \cdots, k_{p}, l_{1}, \cdots, l_{q}\right\}=\{1, \cdots, N\}$. In the latter cases, $\operatorname{dim} \mathfrak{b}\left(k_{1}, \cdots, k_{p} ; l_{1}, \cdots, l_{q} ; \rho_{(m)}\right)=\operatorname{dim} \gamma_{(m)+2}(\Gamma)$, where $\gamma_{(m)+2}(\Gamma)$ is the space of holomorphic $\Gamma$-automorphic forms $f(z)$ transforming by

$$
f(\gamma z)=\pi_{k=1}^{N}\left(c_{k} z^{k}+d_{k}\right)^{m_{k}+2} f(z)
$$

if $\gamma=\left(\gamma_{1}, \cdots, \gamma_{N}\right) \in \Gamma$ and $\gamma_{k}=\left(\begin{array}{ll}a_{k} & b_{k} \\ c_{k} & d_{k}\end{array}\right), k=1, \cdots, N$.

The coordinate vector $\omega^{\circ}$ of $\omega \in \mathfrak{h}\left(k_{1}, \cdots, k_{p} ; l_{1}, \cdots, l_{q} ; \rho_{(m)}\right)$ takes 
values in $F_{(m)} \otimes p_{-}^{k_{1}} \wedge \cdots \wedge p^{k_{p}} \otimes p_{+}^{l_{1}} \wedge \cdots \wedge p_{+}^{l_{p}}$; note that $\boldsymbol{p}_{-}^{k_{1}} \wedge \cdots \wedge \boldsymbol{p}_{-}^{k_{p}}=$ $p^{-\left\langle\phi_{s}\right\rangle}$ for the $S \in W^{\prime}$ acting nontrivially on the $k_{1}, \cdots, k_{p}$ th factors and trivially on the other factors. Similarly, $p_{+}^{l_{1}} \wedge \cdots \wedge p_{+}^{l_{q}}$ is a $Q_{\langle\phi r\rangle}, T \in$ $W^{\prime}$. A $G$-module isomorphism $F_{(m)} \rightarrow F_{1}\left(\Lambda_{(m)}\right) \subset \Lambda^{b}\left(F_{\mathrm{c}}\right)^{*}$ (notations as in $\S 3)$ induces isomorphisms $\mathscr{H}^{a}\left(X, \Gamma, \rho_{(m)}\right) \cong V_{1}\left(\Lambda_{(m)}\right)$ taking $\mathfrak{h}\left(k_{1}, \cdots, k_{p} ; l_{1}, \cdots, l_{a-p} ; \rho_{(m)}\right)$ onto $V_{1}\left(\Lambda_{(m)}\right) \cap \mathscr{H}^{\{a, b\}}\left(\Lambda_{(m)}, \mu, S, T\right)$.

In this case, $\mu, S$, and $T$ are related by: $\mu=S \Lambda_{(m)}=$ $-T \Lambda_{(m)}$. (When $\mathfrak{h}\left(k_{1}, \cdots, k_{p} ; l_{1}, \cdots, l_{q} ; \rho_{(m)}\right) \neq 0$, the condition $S \Lambda_{(m)}=$ $R_{1}^{-1} T \Lambda_{(m)}^{\prime}$ is automatically satisfied.) Since $\rho \sim i d \otimes \cdots \otimes i d$ and since $\rho$ induces a holomorphic mapping on $X$, the condition (6) of $\$ 3$ is satisfied. In view of Proposition 4 of $\$ 3$ and the preceding discussion, one has

Proposition 5. $\mathscr{H}^{a_{1} a_{2} b_{1} b_{2}}=0$ unless $a_{1}+a_{2}=N$ or $a_{1}=a_{2}$ and $b_{1}=$ $b_{2}$. Furthermore, from Theorem 1 and Proposition 5 we obtain

Proposition 6. $\quad \mathscr{H}^{(p, p)}(V)=\Sigma_{a+b=p} \mathscr{H}^{\{2 a, 2 b\}}(V)_{\mathbf{c}}+\sum \mathscr{H}^{a, N-a, p-a, p-N+a}$ where the second summation is taken over integers a with $\min \{a, N-a, p-a, p-N+a\} \geqq 0$. In particular, if $p \leqq N / 2$,

$$
\mathscr{H}^{(p, p)}(V)=\sum_{a+b=p} \mathscr{H}^{\{2 a, 2 b\}}(V)_{c}
$$

In Case $N=1$, one can prove more. The irreducible representations occuring in $\rho^{(b)}$ are the $\rho_{m}$ with $m \equiv b \bmod (2)$ and $0 \leqq m \leqq$ b. Also, $W=W^{\prime} \cong\{ \pm 1\}$. By Proposition $4, \mathscr{H}^{0,1, p, p-1}$ is a sum of subspaces $\mathscr{H}^{\{1,2 p-1\}}\left(\Lambda_{m}, \mu, S, T\right)$ and each such nonzero subspace must satisfy the conditions $n(S)=0, n(T)=1, \mu=s \Lambda_{m}=-T \Lambda_{m}$, and $\mu(Z)=[(p-1)-p] i / 2=-i / 2$. But $S=1, T=-1$ and so $\mu(Z)=$ $\Lambda_{m}(Z)=m i / 2$, with $m \geqq 0$. Thus $\mathscr{H}^{0,1, p, p-1}=0$. Similarly, $\mathscr{H}^{1,0, p-1, p}=$ 0 . This gives

THEOREM 2. In Case $N=1, \mathscr{H}^{(p, p)}(V)=\mathscr{H}^{\{0,2 p\}}(V)_{\mathrm{c}}+\mathscr{H}^{\{2,2(p-1)\}}(V)_{\mathrm{c}}$.

Application 1. Since the subspaces $\mathscr{H}^{\{a, b\}}(V)$ are defined over $\mathbf{Q}$, (see [1], [8]) $\mathscr{H}^{(p, p)}(V)$ is defined over $\mathbf{Q}$ when $p \leqq N / 2$.

Application 2. The Hodge conjecture for $V$ a fiber variety over an algebraic curve attached to an indefinite quaternion algebra over $\mathbf{Q}$. see [2]. 


\section{REFERENCES}

1. R. Hall, Thesis, SUNY at Stony Brook, 1972.

2. R. Hall and M. Kuga, Algebraic cycles in a fiber variety, Scientific Papers of the College of General Education, Univ. of Tokyo, Vol. 25, No. 1 (1975), 1-16.

3. M. Kuga, Fiber varieties over a symmetric space whose fibers are abelian varieties, I, II, Univ. of Chicago Lecture Notes, 1963-64.

4. —, Fibred variety over a symmetric space whose fibers are abelian varieties, Proc. of U.S.-Japan Sem. in Diff. Geom., Kyoto, Japan, (1965), 72-81.

5. Y. Matsushima and S. Murakami, Cohomology groups of vector valued forms on symmetric spaces, Univ.' of Chicago Lecture Notes, Summer 1966.

6. - On vector-bundle valued harmonic forms on symmetric riemannian manifolds, Ann. of Math., 78 (1963), 365-416.

7. Y. Matsushima and G. Shimura, Vector valued differential forms on the product of upper half planes, Ann. of Math., 78 (1963), 417-449.

8. I. Satake, Mountjoy's abelian variety attached to a holomorphic immersion, Number Theory, Alg., Geom., and Comm. Alg., in honor of Y. Akizuki, Kinokuniya, Toyko, 1973, 45-69.

Received February 12, 1976 and in revised form June 17, 1977.

State University of New York at Albany,

Albany, NY 12222 


\section{PACIFIC JOURNAL OF MATHEMATICS}

\section{EDITORS}

RICHARD ARENS (Managing Editor)

University of California

Los Angeles, CA 90024

R. A. BeAumont

University of Washington

Seattle, WA 98105

C. C. MOORE

University of California

Berkeley, CA 94720
J. DUGUNDJI

Department of Mathematics

University of Southern California

Los Angeles, CA 90007

R. FINN AND J. MILGRAM

Stanford University

Stanford, CA 94305

\section{ASSOCIATE EDITORS}
E. F. BECKENBACH
B. H. NEUMANN
F. WOLF
K. YoshidA

\section{SUPPORTING INSTITUTIONS}

UNIVERSITY OF BRITISH COLUMBIA

UNIVERSITY OF SOUTHERN CALIFORNIA

CALIFORNIA INSTITUTE OF TECHNOLOGY

STANFORD UNIVERSITY

UNIVERSITY OF CALIFORNIA

UNIVERSITY OF HAWAII

MONTANA STATE UNIVERSITY

UNIVERSITY OF TOKYO

UNIVERSITY OF NEVADA

UNIVERSITY OF UTAH

NEW MEXICO STATE UNIVERSITY

OREGON STATE UNIVERSITY

UNIVERSITY OF OREGON

OSAKA UNIVERSITY

WASHINGTON STATE UNIVERSITY

UNIVERSITY OF WASHINGTON

AMERICAN MATHEMATICAL SOCIETY

The Supporting Institutions listed above contribute to the cost of publication of this Journal, but they are not owners or publishers and have no responsibility for its contents or policies.

Mathematical papers intended for publication in the Pacific Journal of Mathematics should be in typed form or offset-reproduced (not dittoed), double spaced with large margins. Underline Greek letters in red, German in green, and script in blue. The first paragraph or two must be capable of being used separately as a synopsis of the entire paper. Items of the bibliography should not be cited there unless absolutely necessary, in which case they must be identified by author and Journal, rather than by item number. Manuscripts, in duplicate, may be sent to any one of the four editors. Please classify according to the scheme of Math. Reviews, Index to Vol. 39. All other communications should be addressed to the managing editor, or Elaine Barth, University of California, Los Angeles, California, 90024.

100 reprints are provided free for each article, only if page charges have been substantially paid. Additional copies may be obtained at cost in multiples of 50 .

The Pacific Journal of Mathematics is issued monthly as of January 1966. Regular subscription rate: $\$ 72.00$ a year (6 Vols., 12 issues). Special rate: $\$ 36.00$ a year to individual members of supporting institutions.

Subscriptions, orders for numbers issued in the last three calendar years, and changes of address should be sent to Pacific Journal of Mathematics, 103 Highland Boulevard, Berkeley, California, 94708.

PUBLISHED BY PACIFIC JOURNAL OF MATHEMATICS, A NON-PROFIT CORPORATION

Printed at Jerusalem Academic Press, POB 2390, Jerusalem, Israel. 


\section{Pacific Journal of Mathematics}

\section{Vol. 75, No. $2 \quad$ October, 1978}

Susan Jane Zimmerman Andima and W. J. Thron, Order-induced

topological properties ................................... 297

Gregory Wade Bell, Cohomology of degree 1 and 2 of the Suzuki groups . . 319

Richard Body and Roy Rene Douglas, Rational homotopy and unique

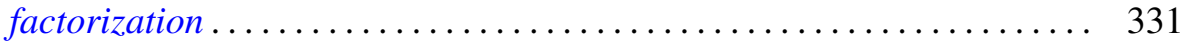

Frank Lewis Capobianco, Fixed sets of involutions ................. 339

L. Carlitz, Some theorems on generalized Dedekind-Rademacher sums .... 347

Mary Rodriguez Embry and Alan Leslie Lambert, The structure of a special class of weighted translation semigroups .....................

Steve Ferry, Strongly regular mappings with compact ANR fibers are

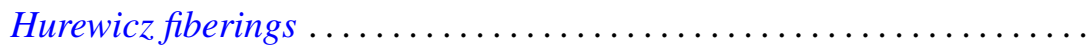

Ivan Filippenko and Marvin David Marcus, On the unitary invariance of the

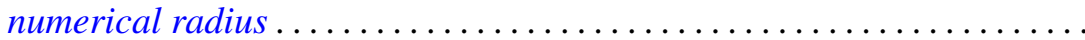

$\mathrm{H}$. Groemer, On the extension of additive functionals on classes of convex

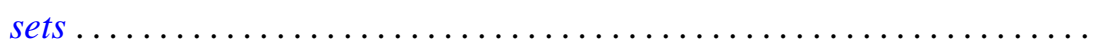

Rita Hall, On the cohomology of Kuga's fiber variety ............... 411

H. B. Hamilton, Congruences on $\mathrm{N}$-semigroups ................. 423

Manfred Herrmann and Rolf Schmidt, Regular sequences and lifting

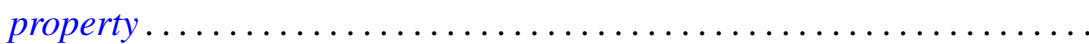

James Edgar Keesling, Decompositions of the Stone-Čech compactification which are shape equivalences .....................

Michael Jay Klass and Lawrence Edward Myers, On stopping rules and the expected supremum of $S_{n} / T_{n}$

Ronald Charles Linton, $\lambda$-large subgroups of $C_{\lambda}$-groups

William Owen Murray, IV and L. Bruce Treybig, Triangulations with the free cell property ............................

Louis Jackson Ratliff, Jr., Polynomial rings and $H_{i}$-local rings ...

Michael Rich, On alternate rings and their attached Jordan rings....

Gary Sampson and H. Tuy, Fourier transforms and their Lipschitz

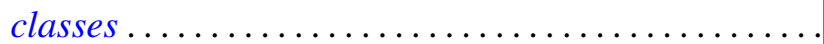

Helga Schirmer, Effluent and noneffluent fixed points on dendrites ...

Daniel Byron Shapiro, Intersections of the space of skew-symmetric maps

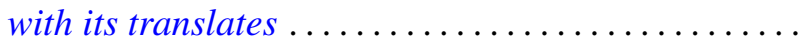

Edwin Spanier, Tautness for Alexander-Spanier cohomology ...

Alan Stein and Ivan Ernest Stux, A mean value theorem for binary digits ...

Franklin D. Tall, Normal subspaces of the density topology . .

William Yslas Vélez, Prime ideal decomposition in $F\left(\mu^{1 / p}\right) \ldots$ 